\title{
Modelica Models for Magnetic Hysteresis, Materials and Transformers
}

\author{
Johannes Ziske, Thomas Bödrich \\ Technische Universität Dresden, Institute of Electromechanical and Electronic Design \\ 01062 Dresden, Germany \\ Johannes.Ziske@tu-dresden.de \\ Thomas.Boedrich@tu-dresden.de
}

\begin{abstract}
Within the Clean Sky project MoMoLib (Modelica Model Library Development for Media, Magnetic Systems and Wavelets) an extension for the Modelica.Magnetic.FluxTubes library has been developed. This extension mainly consists of new flux tubes elements for the consideration of the magnetic hysteresis in the transient simulation of electromagnetic networks, a materials library with hysteresis data of various magnetic materials and a new components package with models of one- and three-phase transformers, which also account for the magnetic hysteresis of the core. This paper briefly presents the implemented hysteresis models for the simulation of the static (ferromagnetic) and dynamic (eddy currents) hysteresis. It shows the accurate computation of the instantaneous hysteresis losses, which becomes increasingly important for the design of electromagnetic components with increasing requirements regarding energy efficiency and mass power densities. Furthermore, the new components of the library extension are introduced and the behavior of the implemented elements is verified and compared to measurements and steel sheet datasheets.
\end{abstract}

Keywords: magnetic hysteresis; Tellinen; Preisach; Modelica.Magneic.Fluxtubes; iron losses;

\section{Introduction}

The Modelica.Magnetic.FluxTubes library was originally developed at the Technische Universität Dresden and has been part of the Modelica Standard Library (MSL) [1] since 2009. The library is based on the well-known concept of magnetic flux tubes $[2,3]$ and allows modeling of magnetic fields with lumped networks. Due to the library elements for modeling of coils, non-linear core material, leakage flux and reluctance forces this library is well-suited for the rough design of electromagnetic components and devices, e.g. actuators, motors, transformers, or hold- ing magnets. Important properties of magnetic components, e.g. saturation behavior, residual magnetism and especially the iron losses are influenced or even determined by the magnetic hysteresis behavior of the involved ferromagnetic materials. Particularly, the consideration of hysteresis losses gets increasingly important during the design process of magnetic components due to increasing requirements on loss power minimization and high mass power densities. Well-known examples of this engineering trend are e.g. the electromobility and more electric aircraft. So far, the ferromagnetic hysteresis is not yet considered in the FluxTubes library. Within a Clean Sky project this issue has been addressed and an extension for the FluxTubes library has been developed. It includes hysteresis elements for the consideration of both static and dynamic hysteresis of ferromagnetic materials. At the Modelica 2012 conference first Modelica hysteresis models have been presented [4]. Now the work on the extension is almost finished and the extended Modelic.Magnetic.FluxTubes library will soon be integrated into the MSL. Two different hysteresis models have been implemented for modeling of static ferromagnetic hysteresis. On the one hand the rather simple but efficient Tellinen model [5], which is characterized by low computational effort and high numerical stability, and on the other hand the more complex but also more accurate and widely accepted Preisach hysteresis model [6]. Dynamic hysteresis is computed by the product of the classical eddy current factor [5, 7], which considers the electrical conductivity and the thickness of magnetic steel sheets, and the time derivative of the magnetic flux density, which causes the formation of eddy currents. To configure the hysteresis models and to adapt them to specific materials the FluxTubes.Material package has also been extended with a package containing hysteresis properties of typical magnetic materials. This data is mainly based on inhouse measurements according to DIN EN 60404-2 using a $25 \mathrm{~cm}$ Epstein frame. With a series of simulations the behavior of the developed hysteresis models has been verified and compared to published 
data of a steel sheet manufacturer as well as to inhouse measurements.

\section{Theory}

This section gives a short introduction to the two implemented static hysteresis models, the Tellinen and the Preisach model, and introduces the approach for consideration of eddy currents and hysteresis losses.

\subsection{The Tellinen Hysteresis Model}

The Tellinen hysteresis model [5] is a comparatively simple model for the description of ferromagnetic (static) hysteresis. Thus, it is easy to implement, numerically stable and fast. Even though the model does not have a magnetic memory, its accuracy is sufficient for a wide range of simulations.

To adapt the model to specific material properties (hysteresis shapes) only the hysteresis envelope curve, i.e. the rising $R(H)$ and falling $F(H)$ branches of the limiting hysteresis loop, must be provided (see Figure 1). Together with their corresponding slope functions $r(H)$ and $f(h)$, which define the slope of $R(H)$ and $F(H)$ with respect to the magnetic field strength $H$, the actual slope $\mathrm{d} B / \mathrm{d} t$ of the current operation point $O(h, b)$ is given by equation (1).

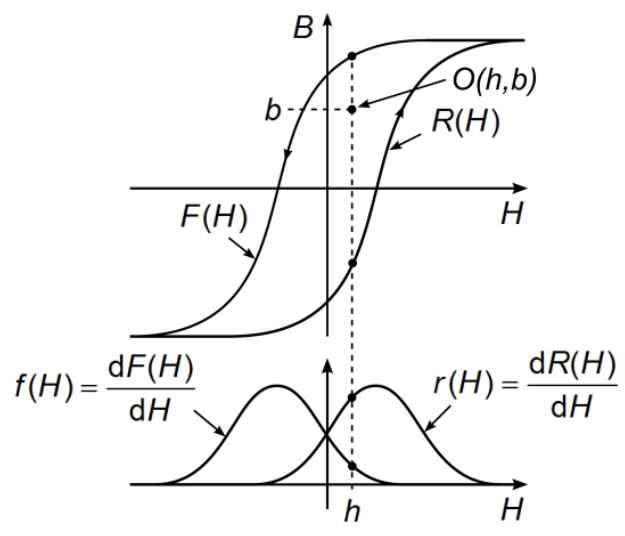

Figure 1: Rising $R(H)$ and falling $F(H)$ branch of the hysteresis envelope curve and their corresponding slope functions $r(H)$ and $f(H)$

$$
\frac{\mathrm{d} B}{\mathrm{~d} t}= \begin{cases}\frac{F(H)-B}{F(H)-R(H)} \cdot r(H) \cdot \frac{\mathrm{d} H}{\mathrm{~d} t} & \text { for } \frac{\mathrm{d} H}{\mathrm{~d} t}>0 \\ \frac{B-R(H)}{F(H)-R(H)} \cdot f(H) \cdot \frac{d H}{\mathrm{~d} t} & \text { for } \frac{\mathrm{d} H}{\mathrm{~d} t}<0\end{cases}
$$

(1) The polarization $j(t)$ of the model is then defined by the integral over all weighted operators outputs multiplied by the saturation polarization $J_{\mathrm{S}}$ : 


$$
j(t)=J_{S} \cdot \iint_{\alpha \geq \beta} P(\alpha, \beta) \cdot \gamma_{\alpha \beta} h(t) \mathrm{d} \alpha \mathrm{d} \beta .
$$

If one splits the Preisach plane into two regions S+ and $S$-, in which all the operators are in the +1 and -1 state, respectively, equation (3) can be simplified to

$$
j(t)=J_{S} \cdot\left(2 \cdot \iint_{S_{+}(t)} P(\alpha, \beta) d \alpha d \beta-1\right) .
$$

It is evident from equation (4) that the Preisach distribution function defines the shape and the behavior of the hysteresis.

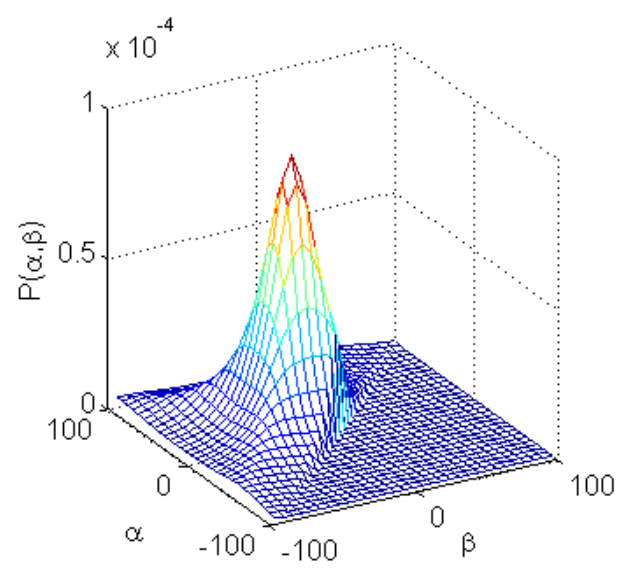

Figure 4: Exemplary Preisach distribution function $P(\alpha, \beta)$ defined over the Preisach plane $(\alpha \geq \beta)$.

Normally, the double integral of $P(\alpha, \beta)$ is not analytically defined. A numerical double integration at every time step of the simulation would be too computationally expensive. In the initial version of the Preisach flux tube element [4] that problem was solved by integrating the Preisach distribution function only once at the start of each simulation for each grid point of a fixed grid over the Preisach plane. The results had been stored in a two-dimensional array (CombiTable2D), which then was used during simulation for table lookup and interpolation to evaluate the integral. The improved version of the element uses now another approach instead, which again improves simulation speed and numerical stability, namely an analytical description of the Everett function [9]. This Everett function $E_{\mathrm{V}}(\alpha, \beta)$ is defined as the integral of $P(\alpha, \beta)$ over the region $R$ :

$$
\begin{aligned}
E_{V}\left(\alpha^{\prime}, \beta^{\prime}\right) & =\iint_{R} P(\alpha, \beta) \mathrm{d} \alpha \mathrm{d} \beta \\
& =\int_{\alpha=\beta^{\prime}} \int_{\beta=\beta^{\prime}}^{\alpha \prime} P(\alpha, \beta) \mathrm{d} \beta \mathrm{d} \alpha .
\end{aligned}
$$

Figure 5 shows the region $\mathrm{R}$ with its integration limits $\alpha$ 'and $\beta$ '.

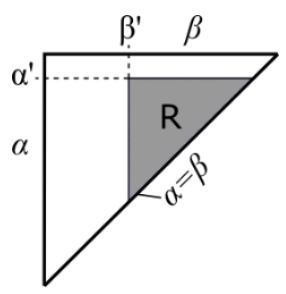

Figure 5: Region $R$ over which the $P(\alpha, \beta)$ is integrated to compute the Everett function $E_{\mathrm{V}}\left(\alpha^{\prime}, \beta^{\prime}\right)$

The use of this Everett function allows the analytical computation of the Preisach model without numerical integration or a table lookup, thus considerably improving the performance of the developed Preisach flux tube model. The implemented analytical form of the Everett function can be adjusted with seven parameters and thus covers only a limited but a wide range of possible hysteresis shapes.

\subsection{Eddy Currents}

In addition to he previously described static hysteresis models this section explains modeling of dynamic hysteresis, i.e. consideration of eddy currents in a ferromagnetic core. An approach proposed in [5] has been implemented. The total magnetic field strength $H(t)$ of an hysteresis element consists of a static $H_{\text {stat }}(t)$ and a dynamic component $H_{\text {eddy }}(t)$ :

$$
H(t)=H_{\text {stat }}(t)+H_{\text {eddy }}(t)
$$

$H_{\text {stat }}(t)$ results from the static Preisach or Tellinen hysteresis model. $H_{\text {eddy }}(t)$ is computed as product of the classical eddy current factor $\sigma_{\mathrm{cl}}[5,7]$ and the change of the magnetic flux density in the core.

$$
H_{\text {eddy }}(t)=\sigma_{c l} \cdot \frac{d B}{d t}
$$

$\sigma_{\mathrm{cl}}$ results from a homogenization approach. It considers the real electrical conductivity $\sigma$ and the thickness of individual steel sheets for computation of an adapted conductivity for a whole stack of laminations:

$$
\sigma_{c l}=\frac{\sigma \cdot d^{2}}{12}
$$

Figure 6 shows exemplarily a simulated hysteresis loop together with its ferromagnetic and its eddy current components. The simulated network model is similar to the one shown in Figure 14. 


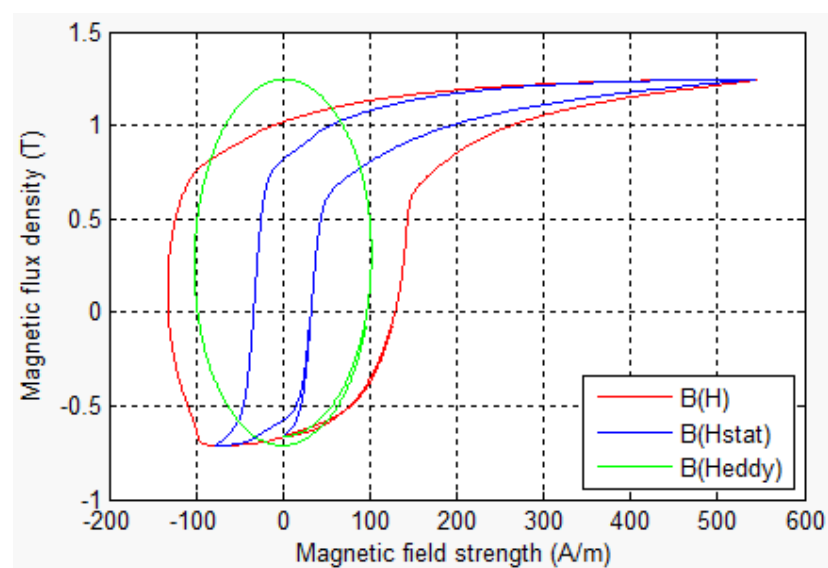

Figure 6: Simulated biased hysteresis loop $B(H)$ with its ferromagnetic component $B\left(H_{\text {stat }}\right)$ and eddy current component $B\left(H_{\text {eddy }}\right)$ for a sinusoidal voltage excitation at $400 \mathrm{~Hz}$

\subsection{Thermal Losses}

Continuous computation of the hysteresis and its static and dynamic components allows for accurate computation of the instantaneous hysteresis losses. The total power loss $P$ consists of the static hysteresis loss $P_{\text {stat }}$ and the eddy current loss $P_{\text {eddy }}$ and can be computed as follows:

$$
P=P_{\text {stat }}+P_{\text {eddy }}=H(t) \cdot \frac{d B(t)}{d t} \cdot V .
$$

$V$ denotes the core volume. For $P_{\text {stat }}$ and $P_{\text {eddy }}$ the equations (10) and (11) apply:

$$
\begin{gathered}
P_{\text {stat }}=H_{\text {stat }}(t) \cdot \frac{d B(t)}{d t} \cdot V \\
P_{\text {eddy }}=H_{\text {eddy }}(t) \cdot \frac{d B(t)}{d t} \cdot V=\sigma_{\mathrm{cl}} \cdot\left(\frac{\mathrm{d} B(t)}{\mathrm{d} t}\right)^{2} \cdot V .
\end{gathered}
$$

According to the hysteresis plot of Figure 6, Figure 7 shows the simulated time courses of the magnetic field strength and the core losses together with their static and dynamic components. The function of the magnetic core as an energy storage is easy to recognize in Figure 7 (b). When the loss power is negative, energy is fed back into the system. Furthermore, the instantaneous computation of the power losses is advantageously, because the losses are computed correctly independent of the applied frequencies and waveforms. The total hysteresis losses can be passed to a conditional heat port as a heat flow. This allows for simple integration of the developed hysteresis elements into a thermal network model.
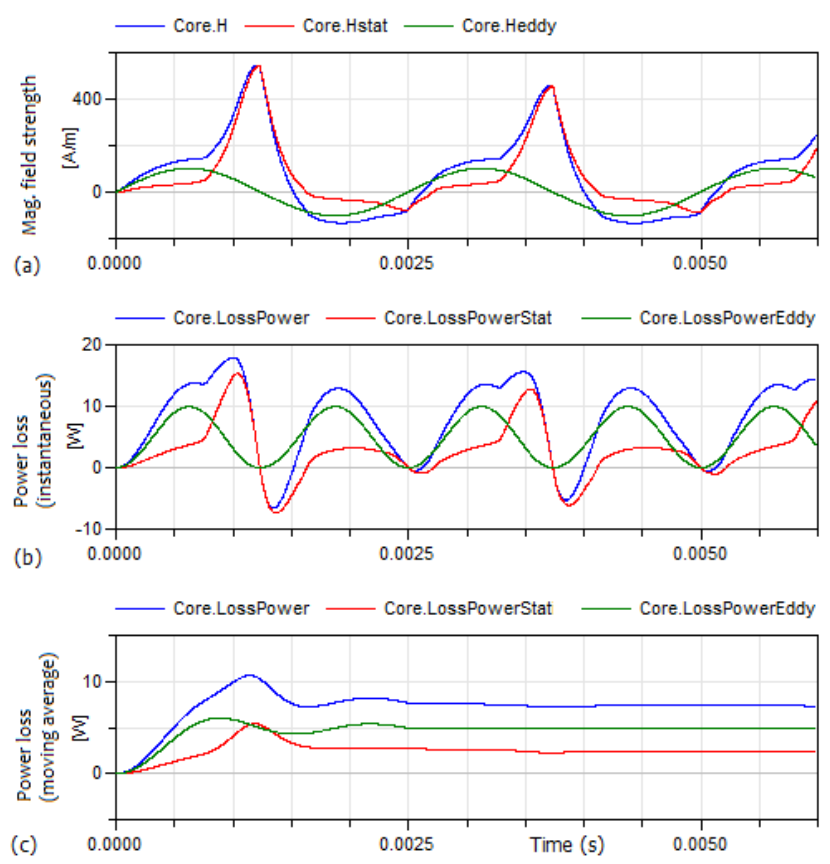

Figure 7: Simulated time course of the magnetic field strength (a), the instantaneous hysteresis losses (b) and the averaged hysteresis losses (c) of a magnetic core element excited with a sinusoidal voltage at $400 \mathrm{~Hz}$ (according to the hysteresis plot of Figure 6).

\section{New Components in the Library Modelica.Magnetic.FluxTubes}

The FluxTubes library extension mainly consists of the developed hysteresis elements for modeling of ferromagnetic materials including permanent magnets, an extended materials package and a new components package with models of one- and threephase transformers.

\subsection{Package HysteresisAndMagnets}

The new hysteresis elements are grouped together in the package FluxTubes.Shapes.HysteresisAndMagnets (see Figure 8). There are four different Tellinen hysteresis models. The difference between these models is the definition of the static hysteresis behavior. The elements CuboidHystTellinenSoft and CuboidHystTellinenHard use very simple hyperbolic tangent shape functions to describe the envelope curve of the hysteresis directly. The shape functions are tailored to the description of soft and hard magnetic materials and can be easily adjusted with four meaningful parameters only (e.g. for the soft magnetic shape functions: saturation magnetization, coercivity, remanence and a multiplier for the vacuum permeability to adjust the slope in the saturation re- 
gion). The elements CuboidHystTellinenEverett and CuboidHystPreisachEverett use the analytical description of the Everett function presented in section 2.2. Several parameter sets to adjust the material behavior are available in the material package HysteresisEverettParameter. An additional option to specify the hysteresis shape offers the element CuboidHysteresisTellinenTable. This element uses table data for the rising and falling branches of the limiting hysteresis loops, and thus allows for the definition of almost arbitrary hysteresis shapes. Two additional elements for modeling permanent magnets are included in the package: the CuboidLinearPermanentMagnet for linear hard magnetic materials defined in Material.HardMagnetic and the CuboidHystTellinenPermanentMagnet which also accounts for hysteresis and thus also for demagnetisation processes of a permanent magnet.

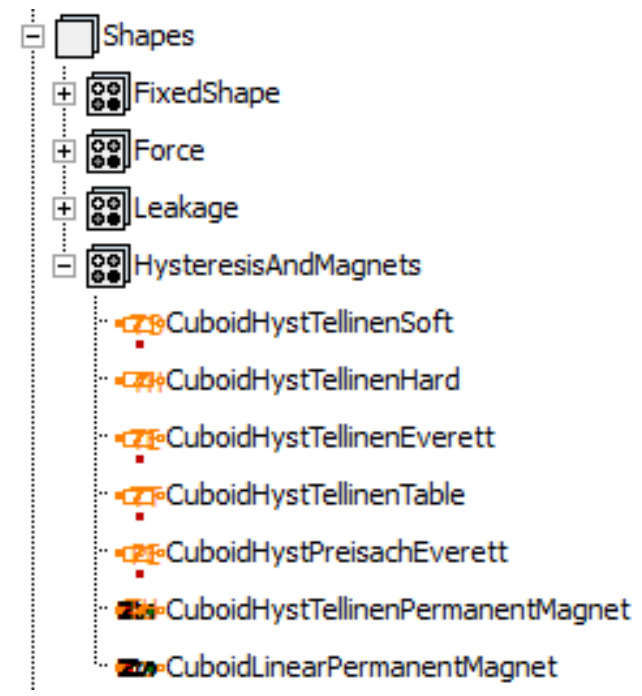

Figure 8: New flux tube elements of package Shapes.HysteresisAndMagnets

Most of these elements also allow for consideration of eddy currents and provide a conditional heat port, which simplifies delivery of the generated hysteresis losses to a thermal network model.

\subsection{Package Components}

Based on the hysteresis elements described above, package Components contains predefined models of single- and three-phase transformers of different topologies (See Figure 9). The transformer models can be widely configured (e.g. core geometry, winding parameters, stray flux, material) and thus easily be adapted to specific needs. Consideration of both static hysteresis and eddy currents allows for accurate computation of losses, saturation effects, inrush currents and frequency behavior. Again, a conditional heat port allows for connection of the magnetic model to a thermal network model. Besides the hysteresis losses also the copper losses of the windings are automatically considered.

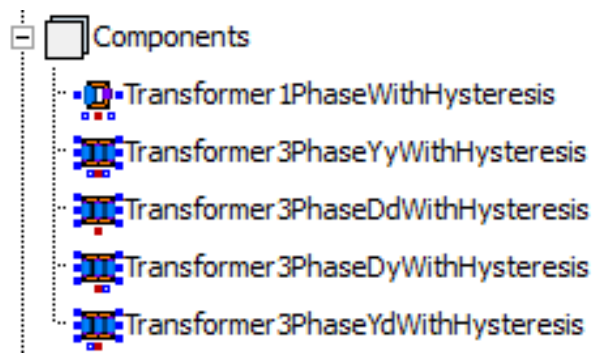

Figure 9: The new Components package of Modelica.Magnetic.FluxTubes.

\subsection{Extension of Package Material}

For a simple adaption of the developed hysteresis models to real magnetic materials two new material packages have been included in the library extension: the package HysteressisEverettParameter and the package HysteresisTableData. Since accurate hysteresis data is hardly available, a measurement setup has been developed and built for hysteresis characterization of magnetic materials according to the DIN EN 60404-2/4/6. Figure 10 shows the block diagram of the utilized measurement setup. The $25 \mathrm{~cm}$ Epstein frame used for the characterization of $30 \mathrm{~mm} \times 280 \mathrm{~mm}$ magnetic steel sheet samples is shown in Figure 11.

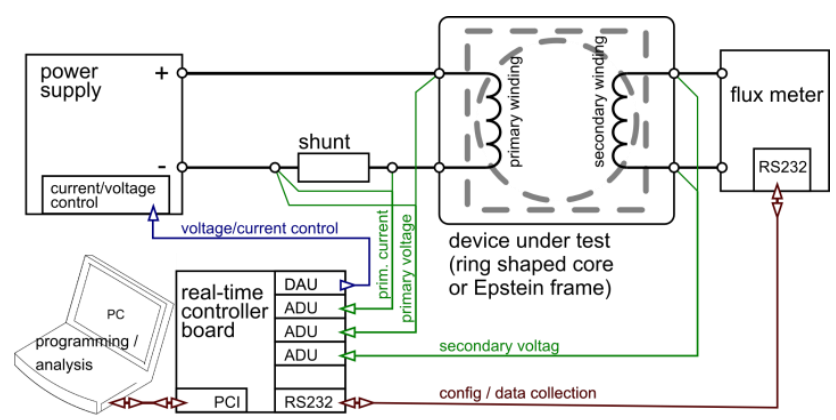

Figure 10: Block diagram of the hysteresis measurement setup

The setup allows hysteresis measurements within a range of the magnetic field strength of $\pm 10 \mathrm{kA} / \mathrm{m}$. For the implementation of package material the static hysteresis characteristic of several steel sheet samples has been determined. Three exemplary hysteresis loops for three different steel sheet qualities are shown in Figure 12. For verification of the developed hysteresis models also dynamic loops have been measured. 


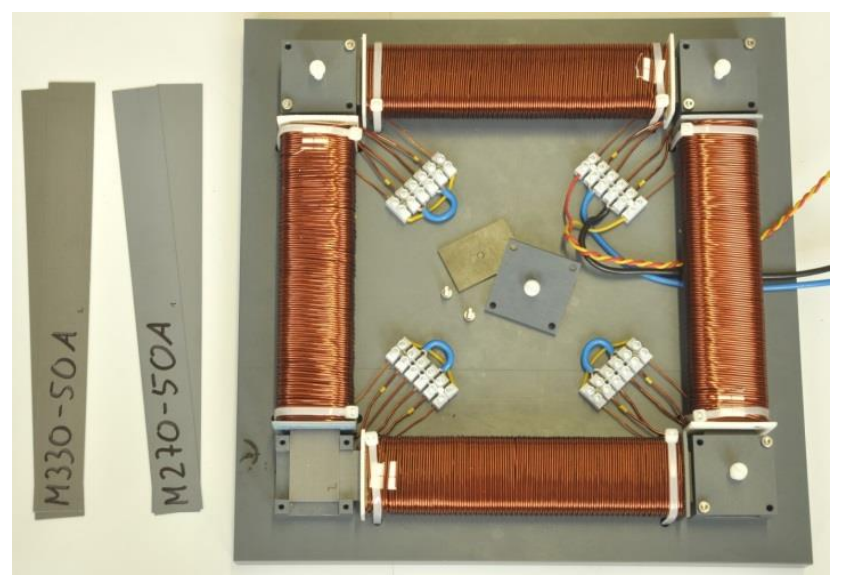

Figure 11: Built-up $25 \mathrm{~cm}$ Epstein frame used for hysteresis characterization of magnetic steel sheets

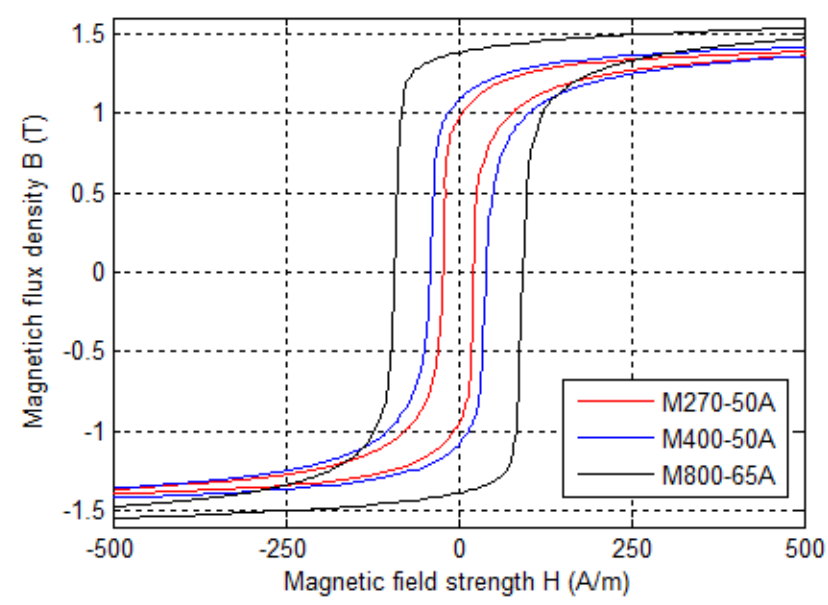

Figure 12: Exemplarily measured hysteresis loops of three different steel sheet qualities

In package HysteresisTableData the measured hysteresis envelope curves are directly stored as table data. The data provided in this package can be used with the CuboidHystTellinenTable hysteresis element. Since the hysteresis elements CuboidHystTellinenEverett and CuboidHystPreisachEverett both need Everett function parameter sets for their configuration (see section 2.2), the package HysteresisEverettParameter contains such predefined parameter sets. They have been identified using the hysteresis loops of package HysteresisTableData as reference. Figure 13 shows the content of package HysteresisTableData. In Addition to the measured hysteresis data some data of cobalt-iron-alloys [10] have also been included in the library. It is planned to extend the material package when new data are available.

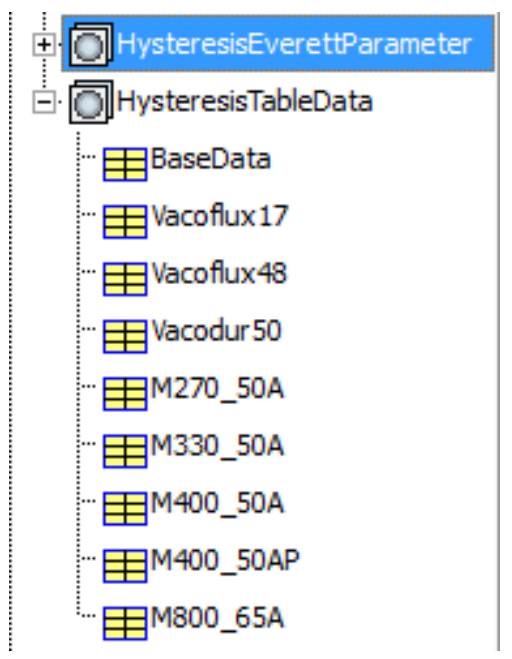

Figure 13: Current content of the hysteresis materials library of Modelica.Magneti.FluxTubes

\section{Verification Results}

To verify the correct simulation of the developed hysteresis models, several experiments have been carried out to compare the model behavior to measurements and to steel sheet datasheets.

\subsection{Static and Dynamic Hysteresis Loops}

In a first experiment the Epstein frame of Figure 11 was used to measure total hysteresis loops (superposition of static and dynamic loops) at several excitation frequencies.

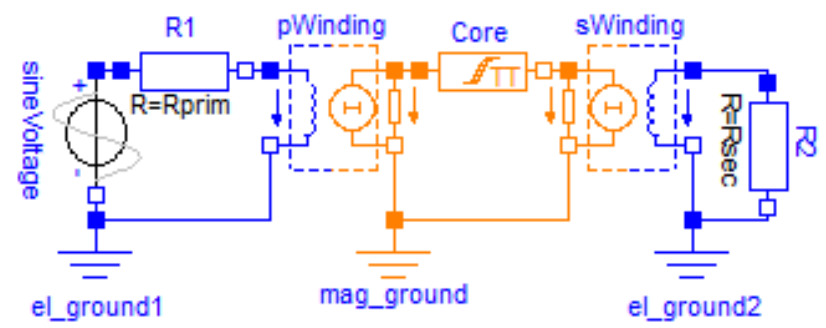

Figure 14: Simple model of the measurement setup including the Epstein frame shown in Figure 11

In addition, the measurement setup has been modeled with a simple model consisting of a sine voltage source, a series resistance, a primary and secondary winding and the magnetic core, latter being modeled with element CuboidHystTellinenTable (see Figure 14). The stray flux of the magnetization coils is considered within the element pWinding. 
Figure 15 shows the measured and the simulated $B(H)$ loops of the magnetic core for identical excitations at several frequencies. In principal, a good agreement can be seen. The deviations in the $200 \mathrm{~Hz}$ loops are likely due to neglection of excess eddy currents, which are not considered in the hysteresis models.

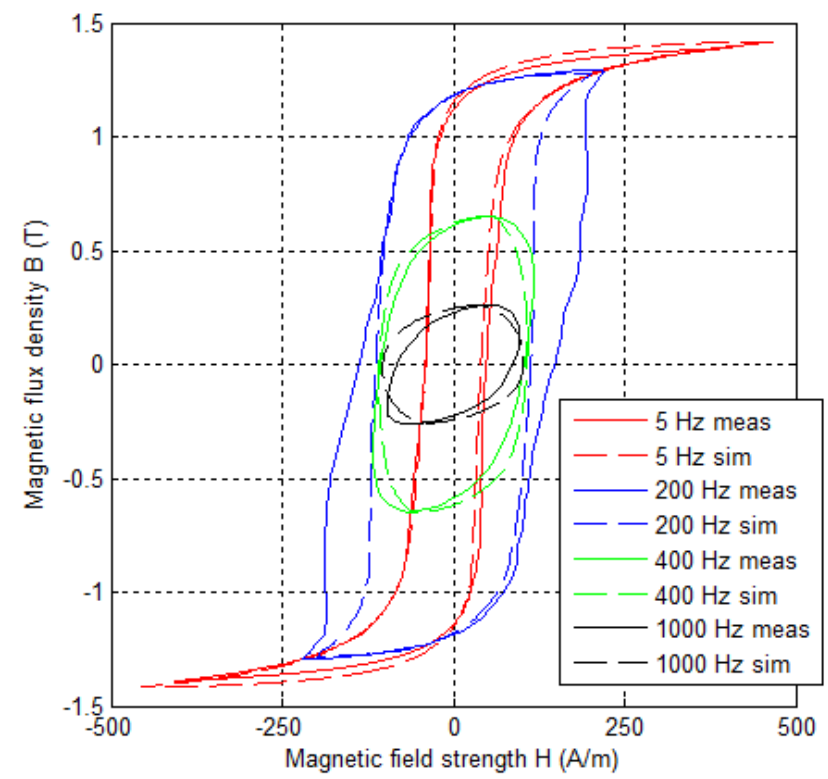

Figure 15: Comparison of measured and simulated total $B(H)$ loops using M330-50A steel sheets and the $\mathrm{Cu}-$ boidHystTellinen hysteresis element for modeling of the core

\subsection{Time Course of Current and Power Losses}
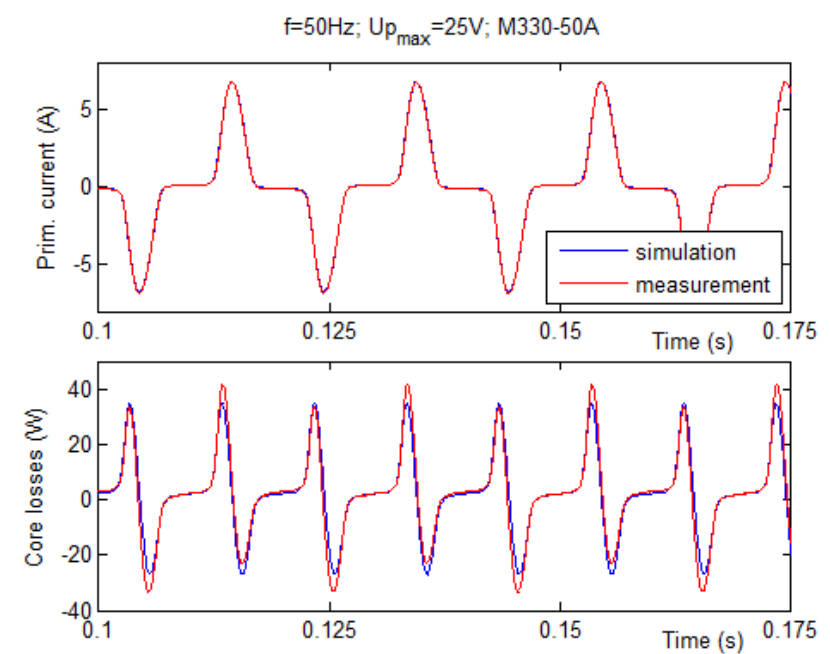

Figure 16: Comparison of measured and simulated primary current and core losses of a transformer excited by a sinusoidal primary voltage of $U_{\mathrm{pmax}}=25 \mathrm{~V}$ and $\mathrm{f}=50 \mathrm{~Hz}$.
In a second experiment a similar setup and model was used to compare the time course of the primary current and the iron losses. This has been done for a highly saturated core at an excitation frequency of $f=50 \mathrm{~Hz}$ and a primary voltage magnitude of $U_{\mathrm{p} \max }=25 \mathrm{~V}$ (see Figure 16) and for a moderately saturated core at $f=100 \mathrm{~Hz}$ and $U_{\mathrm{pmax}}=40 \mathrm{~V}$ (Figure 17).
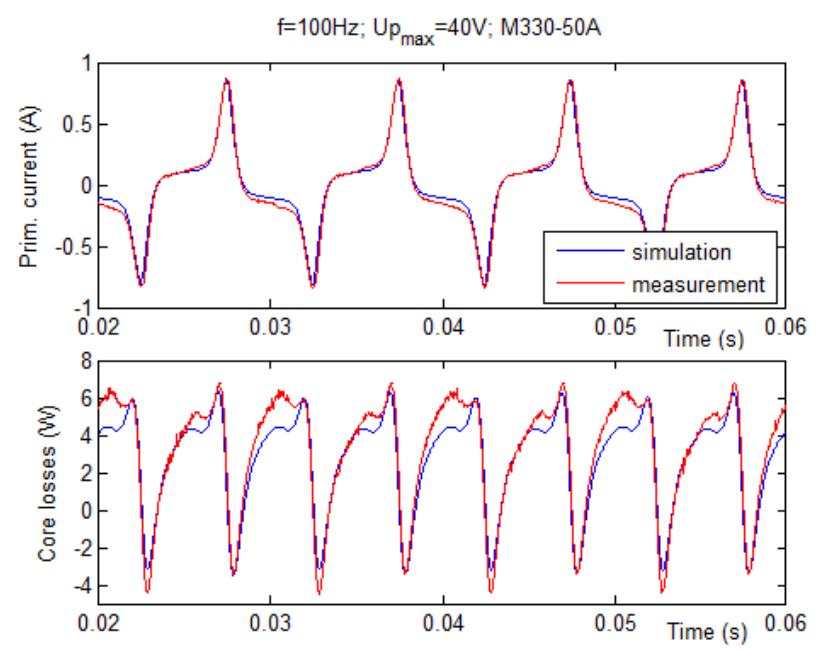

Figure 17: Comparison of measured and simulated primary current and core losses of a transformer excited by a sinusoidal primary voltage $U_{\mathrm{pmax}}=40 \mathrm{~V}$ and a frequency $\mathrm{f}=100 \mathrm{~Hz}$

\subsection{Specific Core Losses}

In a third experiment the model of Figure 14 was used again. The sinusoidal primary voltage has been gradually increased at a fixed frequency of $50 \mathrm{~Hz}$ for two different core materials. For each step the peak value of the magnetic flux density as well as the average power loss of the core have been recorded. Based on the average power loss, the core volume and the density of the core material the specific total losses of the core material have been evaluated. Figure 18 shows the comparison of these values to manufacturer data, which was extracted from the manufacturer datasheets of the investigated materials [11, 12]. Again, a very good agreement over a wide range of magnetic excitations up to saturation is evident.

\section{Summary}

Within the Clean Sky project MoMoLib an extension of the library Modelica.Magnetic.FluxTubes has successfully been implemented. The extension mainly consists of hysteresis elements for modeling of ferromagnetic and dynamic hysteresis of magnetic 


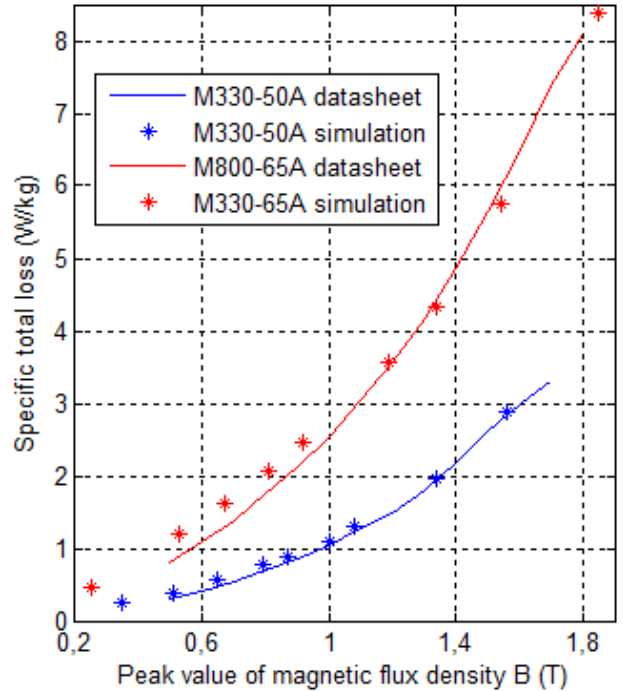

Figure 18: Comparison of specific core losses between data extracted from manufacturer datasheets $[11,12]$ and simulation results for two different steel sheet qualities M330-50A and M800-65A

materials during transient simulation of electromagnetic components with lumped network models. Two different hysteresis models, the Tellinen and the Preisach model have been implemented. Their static hysteresis characteristics can be parameterized in three different ways: with simple $\tanh ()$ shape functions, hysteresis table data or with parameter sets of an analytical Everett function. A material package, mainly based on in-house measurements, provides the hysteresis data of several magnetic materials for easy parameterization of the models. Dynamic hysteresis is considered with a $\mathrm{d} B / \mathrm{d} t$ term. The consideration of static and dynamic hysteresis during transient simulation allows for accurate determination of hysteresis losses. This becomes more and more important during design of electromagnetic actuators and systems due to increasing requirements in terms of power density and miniaturization. A series of experiments showed the correct behavior and the accuracy of the developed hysteresis elements. Additionally, based on the hysteresis elements, models for permanent magnets as well as single- and threephase transformer models have been implemented.

\section{Acknowledgement}

The authors would like to thank the Clean Sky Joint Technology Initiative for funding of the presented work within Project No. 296369 MoMoLib "Modelica Model Library Development for Media, Magnetic Systems and Wavelets". Additionally, the authors wish to thank Prof. Martin Otter of the German
Aerospace Center (DLR) for his valuable support regarding numerical efficiency of the developed hysteresis models.

\section{References}

[1] Modelica Association, Modelica Standard Library, https://www.modelica.org/libraries/-Modelica (May 11, 2012).

[2] T. Bödrich and T. Roschke, A Magnetic Library for Modelica, in Proc. of the 4th International Modelica Conference, 2005, pp. 559-565.

[3] T. Bödrich, Electromagnetic Actuator Modelling with the Extended Modelica Magnetic Library, Proc. of 6th Int. Modelica Conf., Bielefeld, Germany, March 3-4, pp. 221-227, 2008.

[4] J. Ziske, Magnetic Hysteresis Models for Modelica, Proc. of 9th Int. Modelica Conf., Munich, Germany, September 3-5, pp. 151-158, 2012

[5] J. Tellinen, A Simple Scalar Model for Magnetic Hysteresis, IEEE Transactions on Magnetics, vol. 24, no. 4, pp. 2200 - 2206, July 1998.

[6] F. Preisach, Über die magnetische Nachwirkung, Zeitschrift für Physik A Hadrons and Nuclei, vol. 94, pp. 277-302, 1935.

[7] A. J. Bergqvist and S. G. Engdahl, A Homogenization Procedure of Field Quantities in Laminated Electric Steel, Magnetics, IEEE Transactions on, vol.37, no.5, pp.3329-3331, Sep 200

[8] I. Mayergoyz, Mathematical Models of Hysteresis and their Application. Elsevier, 2003.

[9] T. Yamaguchi and F. Ueda and E. Yamamoto, Simulation of Hysteresis Characteristics of Core Materials Using the Everett Function, Magnetics, IEEE Transactions on , vol. 4, no.6, pp.353-359, Jun 1989

[10] Soft Magnetic Cobalt-Iron-Alloys, Vacuumschmelze GmbH, 2001, http://www.vacuumschmelze.com/fileadmin/docroot/medialib/documents/broschue-ren/htbrosch/Pht-004_e.pdf (May 5th, 2013).

[11] ThyssenKrupp Steel AG: Power Core M800-65A, 2009. http://www.thyssenkrupp-steel-europe.com/upload/binarydata_tksteel05d4cms/59/90/78/02/00/00/2789059/Werkstoffblatt_M800-65A.pdf (Dec. $\left.6^{\text {th }}, 2013\right)$

[12] ThyssenKrupp Steel AG: Power Core M330-50A, 2009. http://www.thyssenkrupp-steel-europe.com/upload/binarydata_tksteel05d4cms/45/90/78/02/00/00/2789045/Werkstoffblatt_M330-50A.pdf (Dec. $\left.6^{\text {th }}, 2013\right)$ 